\title{
The Equational Theory of Prebisimilarity over Basic CCS with Divergence $^{\star}$
}

\author{
Luca Aceto $^{\mathrm{a}, *}$, Silvio Capobianco ${ }^{\mathrm{a}}$, Anna Ingolfsdottir ${ }^{\mathrm{a}}$, Bas Luttik $^{\mathrm{b}}$ \\ a School of Computer Science, Reykjavik University, Iceland \\ ${ }^{\mathrm{b}}$ Department of Mathematics and Computer Science, Technische Universiteit Eindhoven, The Netherlands
}

\begin{abstract}
This paper studies the equational theory of prebisimilarity, a bisimulation-based preorder introduced by Hennessy and Milner in the early 1980s, over basic CCS with the divergent process $\Omega$. It is well known that prebisimilarity affords a finite ground-complete axiomatization over this language; this study proves that this ground-complete axiomatization is also complete in the presence of an infinite set of actions. Moreover, in sharp contrast to this positive result, it is shown that prebisimilarity is not finitely based over basic CCS with the divergent process $\Omega$ when the set of actions is finite and non-empty.
\end{abstract}

Key words:

PACS:

\section{Introduction}

The notion of prebisimilarity $[5,9,13,17]$, or bisimulation preorder, has played a key role in the study of domain-theoretic and term models for Milner's CCS [9] and related languages $[1,3,7]$. In particular, as shown in [4], Abramsky's classic domaintheoretic, synchronization-tree-based model for bisimilarity (presented in [1]) is fully abstract with respect to (the finitary part of) prebisimilarity for a natural class of GSOS languages [6]. This means that two processes specified in any GSOS language satisfying the restrictions considered in [4] are related by (the finitary part of) prebisimilarity if, and only if, their denotations are similarly related within

\footnotetext{
‡ The first three authors were partly supported by the project "The Equational Logic of Parallel Processes" (nr. 060013021) of The Icelandic Research Fund.

* Corresponding author.

Email addresses: luca@ru.is (Luca Aceto), silvio@ru.is (Silvio Capobianco), annai@ru.is (Anna Ingolfsdottir), s.p.luttik@tue.nl (Bas Luttik).
}

Preprint submitted to Elsevier
Abramsky's denotational model. This behavioural semantics for processes is therefore in complete agreement with the denotational semantics, thus achieving results for concurrent processes modulo a bisimulation-like behavioural semantics that are akin to those presented in the classic papers $[12,15]$.

The simplest process language over which prebisimilarity can be naturally defined is basic CCS extended with the divergent process $\Omega$. We refer to this language as $\mathrm{BCCS}_{\Omega}$ in what follows. This is a language that contains only the basic process algebraic operators from CCS [14], but is sufficiently powerful to express all finite synchronization trees in Abramsky's domain. Intuitively, $\Omega$ is the process about whose behaviour we have no information whatsoever, and is essentially a syntactic representation of the least element in Abramsky's domain.

In this paper, we study the equational theory of prebisimilarity over the language $\mathrm{BCCS}_{\Omega}$. It is well known that prebisimilarity affords a finite groundcomplete axiomatization over this language (see, 
e.g., [7]). This finite axiomatization is obtained by adding the inequation

$$
\Omega \preccurlyeq x
$$

to the complete axiomatization of bisimilarity over basic CCS [14]. Ground-complete axiomatizations for prebisimilarity over languages that extend $\operatorname{BCCS}_{\Omega}$ are usually obtained by reduction to the ground-complete axiomatization for $\mathrm{BCCS}_{\Omega}$ (see, e.g., $[3,4,7])$, and typically require the use of axiom schemas [2]. We are not aware of any study of complete axiomatizations for prebisimilarity over $\mathrm{BCCS}_{\Omega}$. We recall that a complete axiomatization for prebisimilarity over $\mathrm{BCCS}_{\Omega}$ is a sound collection of inequations that allows us to prove exactly all the valid inequalities between terms that may possibly contain variables.

The aim of this paper is to study the full equational theory of prebisimilarity over $\mathrm{BCCS}_{\Omega}$. Our first main result shows that, in the presence of an infinite set of actions, the classic ground-complete axiomatization for prebisimilarity over $\mathrm{BCCS}_{\Omega}$ is complete (Theorem 3). In sharp contrast to this positive result, we then show that prebisimilarity is not finitely based over $\operatorname{BCCS}_{\Omega}$ when the set of actions is finite and non-empty (Theorem 11). We prove this negative result by exhibiting an infinite family of sound inequations whose elements cannot all be proved to hold from any finite sound axiom system.

The paper is organized as follows. Section 2 presents preliminaries on the language $\mathrm{BCCS}_{\Omega}$, its operational semantics and prebisimilarity. We then show that, in the presence of an infinite collection of actions, prebisimilarity affords a finite, complete, inequational axiomatization over $\mathrm{BCCS}_{\Omega}$ (Section 3 ). We conclude the paper by proving that, in contrast to that result, prebisimilarity is not finitely based when the collection of actions is finite and non-empty (Section 4).

\section{Basic CCS with divergence and prebisimilarity}

The language $\mathrm{BCCS}_{\Omega}$ is a basic process algebra for expressing finite process behaviour. Its syntax consists of closed (process) terms $p, q$ that are constructed from constants $\mathbf{0}$ and $\Omega$, a binary operator _+ _ called alternative composition, and unary prefix operators $a_{-}$, where $a$ ranges over some non-empty set $A$ of actions (with typical elements $a, b$ ). Intuitively, as we shall make precise in what follows, $\mathbf{0}$ stands for a process that has no behaviour whatsoever. On the other hand, $\Omega$ denotes a process whose behaviour is unknown. (Readers familiar with denotational semantics may think of $\Omega$ as denoting the least element in a domain, that is, as a point with no information.)

Open terms $t, u, l, r$ can moreover contain occurrences of variables from a countably infinite set $V$ (with typical elements $x, y, z$ ).

In what follows, for each $n \geq 0$, we use $a^{n} \mathbf{0}$ to stand for the term $\mathbf{0}$ if $n=0$, and for $a\left(a^{n-1} \mathbf{0}\right)$ if $n>0$.

A (closed) substitution maps variables in $V$ to (closed) terms. For every term $t$ and substitution $\sigma$, the term $\sigma(t)$ is obtained by replacing every occurrence of a variable $x$ in $t$ by $\sigma(x)$. Note that $\sigma(t)$ is closed if $\sigma$ is a closed substitution.

The operational semantics of the language $\mathrm{BCCS}_{\Omega}$ is given by two ingredients: a family of transition relations $\stackrel{a}{\longrightarrow}(a \in A)$ and a convergence predicate $\downarrow$.

Intuitively, closed $\mathrm{BCCS}_{\Omega}$ terms represent finite process behaviours, where $\mathbf{0}$ and $\Omega$ do not exhibit any behaviour, $p+q$ is the nondeterministic choice between the behaviours of $p$ and $q$, and $a p$ executes action $a$ to transform into $p$. This intuition is captured, in the style of Plotkin [16], by the transition rules below, which give rise to $A$-labelled transitions between closed terms.

$$
\stackrel{x \stackrel{a}{\longrightarrow} x^{\prime}}{a x \stackrel{a}{\longrightarrow} x} \quad \frac{y \stackrel{a}{\longrightarrow} y^{\prime}}{x+y \stackrel{a}{\longrightarrow} y^{\prime}}
$$

The convergence predicate $\downarrow$ is the least predicate over closed $\mathrm{BCCS}_{\Omega}$ terms satisfying the rules given below.

$$
\overline{\mathbf{0} \downarrow} \quad \overline{a p \downarrow} \frac{p \downarrow}{p+q \downarrow}
$$

Intuitively, the above predicate holds for those terms whose initial behaviour is completely specified, with the understanding that the behaviour of $\Omega$ is unspecified. So, for instance, $a \Omega \downarrow$, but neither $\Omega \downarrow$ nor $a \mathbf{0}+\Omega \downarrow$ hold.

The operational semantics is extended to open terms by assuming that variables afford no transitions and that $x \downarrow$ holds, for each variable $x$.

\section{Definition 1}

(i) For each $B C C S_{\Omega}$ term $t$, we write $\mathcal{I}(t)$ for the collection of initial actions of $t$, namely $\{a \mid$ $t \stackrel{a}{\longrightarrow} t^{\prime}$, for some $\left.t^{\prime}\right\}$.

(ii) For $B C C S_{\Omega}$ terms $t, t^{\prime}$ and sequence of actions $a_{1} \ldots a_{k}$ with $k \geq 0$, we write $t \stackrel{a_{1} \ldots a_{k}}{\longrightarrow} t^{\prime}$ iff there is a sequence of terms $t_{0}, \ldots, t_{k}$ such that 


$$
t=t_{0} \stackrel{a_{1}}{\longrightarrow} t_{1} \stackrel{a_{2}}{\longrightarrow} \cdots t_{k-1} \stackrel{a_{k}}{\longrightarrow} t_{k}=t^{\prime} .
$$

(iii) For $B C C S_{\Omega}$ terms $t, t^{\prime}$ and $k \geq 0$, we write $t \rightarrow^{k} t^{\prime}$ iff $t \stackrel{a_{1} \ldots a_{k}}{\longrightarrow} t^{\prime}$ for some sequence of actions $a_{1} \ldots a_{k}$.

(iv) The depth of a BCCS $S_{\Omega}$ term $t$, written depth $(t)$, is the largest $k$ such that $t \rightarrow{ }^{k} t^{\prime}$ holds for some $t^{\prime}$.

The following notion of prebisimilarity is a relevant notion of behavioural preorder in the presence of divergence as adopted in, e.g., $[1,3,4,7,13]$. We refer the interested reader to those papers and the references therein for a wealth of results on the semantic theory of $\mathrm{BCCS}_{\Omega}$ and related languages modulo prebisimilarity.

Definition 2 The relation $\preceq$ is the largest relation over closed $B C C S_{\Omega}$ terms satisfying the following clauses, whenever $p \preceq q$,

(i) for each $p^{\prime}$, if $p \stackrel{a}{\longrightarrow} p^{\prime}$ then there exists a $q^{\prime}$ such that $q \stackrel{a}{\longrightarrow} q^{\prime}$ and $p^{\prime} \preceq q^{\prime}$;

(ii) if $p \downarrow$, then

(a) $q \downarrow$ and

(b) for each $q^{\prime}$, if $q \stackrel{a}{\longrightarrow} q^{\prime}$ then there exists a $p^{\prime}$ such that $p \stackrel{a}{\longrightarrow} p^{\prime}$ and $p^{\prime} \preceq q^{\prime}$.

The relation $\preceq$ is a preorder that is compatible with prefixing and alternative composition, i.e., it is a precongruence over closed $\mathrm{BCCS}_{\Omega}$ terms; it is generalized to open terms in the standard way by stipulating that $t \preceq u$ holds for terms $t$ and $u$ iff $\sigma(t) \preceq$ $\sigma(u)$ for each closed substitution $\sigma$.

\section{A finite basis result for $\mathrm{BCCS}_{\Omega}$ over an infinite set of actions}

It is well known that the following finite axiom system $\mathcal{E}$ is sound and ground-complete for $\preceq$ over $\operatorname{BCCS}_{\Omega}[7]$. (Below we write $t \approx u$ as a short-hand for the pair of inequations $t \preccurlyeq u$ and $u \preccurlyeq t$.)

$$
\begin{array}{rlrl} 
& \text { A1 } & x+y & \approx y+x \\
\text { A2 } & (x+y)+z & \approx x+(y+z) \\
\text { A3 } & x+x & \approx x \\
\text { A4 } & x+\mathbf{0} & \approx x \\
\text { O } & \Omega & \preccurlyeq x
\end{array}
$$

This means that all the inequalities between closed $\operatorname{BCCS}_{\Omega}$ terms that are valid modulo $\preceq$ can be proved from the axiom system above using the standard rules of (in)equational logic; see, e.g., the textbook [8].
In the remainder of this paper, process terms are considered modulo A1-A4. A term $x, a t^{\prime}$ or $\Omega$ is a summand of a term $t$ if there exists a term $u$ such that $t=x+u, t=a t^{\prime}+u$ or $t=\Omega+u$, respectively. We use summation $\sum_{i=1}^{n} t_{i}$ (with $n \geq 0$ ) to denote $t_{1}+\cdots+t_{n}$, where the empty sum denotes $\mathbf{0}$. As binding convention, alternative composition and summation bind more weakly than prefixing. Modulo the equations $\mathrm{A} 1-\mathrm{A} 4$ each $\mathrm{BCCS}_{\Omega}$ term $t$ can be written in the form $\sum_{i=1}^{n} t_{i}[+\Omega]$, where each $t_{i}$ is either a variable or is of the form $a t^{\prime}$ for some action $a$ and term $t^{\prime}$. The notation $[+\Omega]$ indicates that $\Omega$ is an optional summand. It is easy to see that $t \downarrow$ holds iff $\Omega$ is not a summand of $t$.

It is natural to wonder whether $\mathcal{E}$ is a finite basis for the collection of inequations that hold in the algebra of closed $\mathrm{BCCS}_{\Omega}$ terms modulo $\preceq$ - that is, whether each valid inequation $t \preccurlyeq u$ can be proved from $\mathcal{E}$. We shall now show that this is indeed the case when the collection $A$ of actions is infinite. This is the import of the following theorem.

Theorem 3 Assume that $A$ is infinite. Let $t, u$ be $B C C S_{\Omega}$ terms. Then $t \preceq u$ iff $t \preccurlyeq u$ can be proved from $\mathcal{E}$.

The rest of this section will be devoted to a proof of the above theorem. Since the soundness of $\mathcal{E}$ is well known, we focus on showing that $\mathcal{E}$ is complete. The following notion of distinguishing substitution will be the crucial tool in our proof.

Definition 4 Let $t$ and $u$ be $B C C S_{\Omega}$ terms. A distinguishing substitution for $t$ and $u$ is a substitution $\sigma$ that maps every variable $x$ to a closed term $a_{x} \mathbf{0}$ $\left(a_{x} \in A\right)$ such that

(i) the action $a_{x}$ associated with the variable $x$ is fresh with respect to $t$ and $u$, i.e., it does not occur in $t$ nor in $u$, and

(ii) the substitution is injective so that $a_{x}=a_{y}$ implies $x=y$ for all variables $x$ and $y$.

Note that distinguishing substitutions exist for $t$ and $u$ provided that the set of actions $A$ is infinite. We shall now establish a special property for distinguishing substitutions from which Theorem 3 will easily follow.

Lemma 5 If $\sigma$ is a distinguishing substitution for $t$ and $u$, then $\sigma(t) \preceq \sigma(u)$ implies $t \preccurlyeq u$.

PROOF. Suppose that $\sigma$ is a distinguishing substitution for $t$ and $u$, and suppose that $\sigma(t) \preceq \sigma(u)$; we prove with induction on the sum of the depths of $t$ and $u$ that $t \preccurlyeq u$.

Note that, modulo A1-A4, we can write 


$$
t=\sum_{i \in I} a_{i} t_{i}+\sum_{j \in J} x_{j}[+\Omega]
$$

and

$$
u=\sum_{k \in K} b_{k} u_{k}+\sum_{\ell \in L} y_{\ell}[+\Omega]
$$

We shall now first argue that $\sigma(t) \preceq \sigma(u)$ implies a correspondence between the summands of $t$ and the summands of $u$.

First consider a summand $a_{i} t_{i}(i \in I)$ of $t$. It gives rise to a transition $\sigma(t) \stackrel{a_{i}}{\longrightarrow} \sigma\left(t_{i}\right)$, which, according to the definition of prebisimilarity, must be matched by a transition from $\sigma(u)$. This means that either there exists $k \in L$ such that $a_{i}=b_{k}$ and $\sigma\left(t_{i}\right) \preceq$ $\sigma\left(u_{k}\right)$, or there exists $\ell \in L$ such that $\sigma\left(y_{\ell}\right) \stackrel{a}{\longrightarrow} u^{\prime}$ with $a_{i}=a$ and $\sigma\left(t_{i}\right) \preceq u^{\prime}$. However, the latter case is excluded by the freshness of the actions assigned to variables by the distinguishing substitution $\sigma$, for $\sigma\left(y_{\ell}\right)=a_{y_{\ell}} \mathbf{0}$ where $a_{y_{\ell}} \neq a_{i}$. So we can conclude that there exists $k \in K$ such that $a_{i}=b_{k}$ and $\sigma\left(t_{i}\right) \preceq \sigma\left(u_{k}\right)$. Moreover, since $\sigma$ is clearly also a distinguishing substitution for $t_{i}$ and $u_{k}$, by the induction hypothesis we can conclude that $t_{i} \preccurlyeq u_{k}$.

Next, we consider a summand $x_{j}(j \in J)$ of $t$. It gives rise to a transition $\sigma(t) \stackrel{a_{x_{j}}}{\longrightarrow} \mathbf{0}$, which, according to the definition of prebisimilarity, must be matched by a transition from $\sigma(u)$. This means that either there exists $k \in L$ such that $a_{x_{j}}=b_{k}$ and $\mathbf{0} \preceq \sigma\left(u_{k}\right)$, or there exists $\ell \in L$ such that $\sigma\left(y_{\ell}\right) \stackrel{a}{\longrightarrow}$ $u^{\prime}$ with $a_{x_{j}}=a$ and $\sigma\left(t_{i}\right) \preceq u^{\prime}$. However, now the former case is excluded by the freshness of the actions assigned to variables by the distinguishing substitution $\sigma$, for $a_{x_{j}} \neq b_{k}$ for all $k \in K$. So we can conclude that there exists $\ell \in L$ such that $a_{x_{j}}=$ $a_{y_{\ell}}$, from which it follows by the injectivity of $\sigma$ that $x_{j}=y_{\ell}$.

We have established that for all $i \in I$ there exists $k \in K$ such that $a_{i}=b_{k}$ and $t_{i} \preccurlyeq u_{k}$, and for all $j \in J$ there exists $\ell \in L$ such that $x_{j}=y_{\ell}$. Hence, modulo A1-A4,

$$
u=u+\sum_{i \in I} a_{i} t_{i}+\sum_{j \in J} x_{j} .
$$

To complete the proof we now distinguish two cases, according to whether $t$ has the optional summand $\Omega$ or not:

CAse 1: Suppose $\Omega$ is a summand of $t$.

Then we can use the axiom $\mathrm{O}$ to replace the $\Omega$ summand of $t$ with $u$ and subsequently apply (1), obtaining

$$
t \preccurlyeq \sum_{i \in I} a_{i} t_{i}+\sum_{j \in J} x_{j}+u \approx u .
$$

CAse 2: Suppose $\Omega$ is not a summand of $t$.

Then $t \downarrow$, and hence $u \downarrow$, so $\Omega$ is not a summand of $u$ either. Clearly, according to (1), $u \approx u+t$, and by a similar reasoning as above it can be established that, modulo A1-A4, $t \approx t+u$. Hence, $t \approx u$.

This completes the proof of the lemma.

To complete the proof of Theorem 3, suppose that $t \preceq u$. By the assumption that $A$ is infinite, there exists a distinguishing substitution $\sigma$ for $t$ and $u$, and from $t \preceq u$ it follows that $\sigma(t) \preceq \sigma(u)$. Hence, by Lemma $5, t \preccurlyeq u$.

Remark 6 Our proof above establishes directly that $\mathcal{E}$ is complete for $\preceq$ over $B C C S_{\Omega}$. An alternative proof takes as a starting point the proof in [7] that $\mathcal{E}$ is ground-complete, and continues with the observation that, since there are no occurrences of actions in the axioms, any derivation from $\mathcal{E}$ of a ground equation $t \approx u$ is still valid if some actions are replaced by variables. Thus, a derivation of an arbitrary sound equation $t \approx u$ can be obtained from a derivation of $t^{\prime} \approx u^{\prime}$, where $t^{\prime}$ and $u^{\prime}$ are ground terms obtained from $t$ and $u$, respectively, by replacing every variable by a fresh action. This technique is, e.g., used in [11] and is the idea underlying Groote's general proof technique of inverted substitutions [10].

\section{Prebisimilarity is not finitely based over a finite set of actions}

In the previous section, we proved that the axiom system $\mathcal{E}$ is complete for prebisimilarity over $\mathrm{BCCS}_{\Omega}$ if the set of actions $A$ is infinite. It follows that the ordered algebra of closed $\mathrm{BCCS}_{\Omega}$ terms modulo prebisimilarity is finitely based in the presence of an infinite collection of actions. In the proof of Theorem 3 we made use of the infinity of $A$ when defining the notion of distinguishing substitution (see Definition 4 . It is therefore natural to wonder whether $\mathcal{E}$ is also complete when the collection of actions is finite. The following example shows that $\mathcal{E}$ is, in fact, incomplete if $A$ is finite.

Example 7 Assume that $A=\{a\}$. It is not hard to see that

$$
a(x+a \Omega)+\Omega \preceq a x+a a \mathbf{0} .
$$


In fact, on the one hand, if $p$ is a closed term of depth zero, then $p+a \Omega \preceq a \mathbf{0}$. On the other hand, if $p \stackrel{a}{\longrightarrow} p^{\prime}$ for some $p^{\prime}$, then $p+a \Omega \preceq p$.

However, the above inequality cannot be derived from $\mathcal{E}$. This follows from the completeness theorem for inequational logic, because the axiom system $\mathcal{E}$ is sound regardless of the cardinality of the set of actions $A$ whereas the above inequality is not sound when $A$ contains at least two actions.

In the remainder of this section, we shall prove that, in sharp contrast to Theorem 3, prebisimilarity is not finitely based over $\mathrm{BCCS}_{\Omega}$ when the set of actions is finite and non-empty. This means that no finite collection of inequations that is sound with respect to prebisimilarity is complete.

We begin by stating two useful lemmas, which will find application in the technical developments to follow.

Lemma 8 Assume that $p$ is a closed term such that $a^{n} \mathbf{0} \preceq p$ for some $n \geq 0$. Then $p \preceq a^{n} \mathbf{0}$.

PROOF. The relation

$$
\left\{\left(p, a^{n} \mathbf{0}\right) \mid n \geq 0 \text { and } a^{n} \mathbf{0} \preceq p\right\}
$$

satisfies the defining clauses of $\preceq$.

Lemma 9 Let $t$ and $u$ be terms and suppose that $t \preceq u$. Then

(i) $\operatorname{depth}(t) \leq \operatorname{depth}(u)$;

(ii) $\mathcal{I}(t) \subseteq \mathcal{I}(u)$; moreover, if $t \downarrow$, then $\mathcal{I}(t)=\mathcal{I}(u)$; and

(iii) if there exists some $t^{\prime}$ such that $t \rightarrow^{k} t^{\prime}$ and $t^{\prime}$ has the variable $x$ as a summand, then there exists some $u^{\prime}$ such that $u \rightarrow^{k} u^{\prime}$ and $u^{\prime}$ has $x$ as a summand; moreover, if $t^{\prime} \downarrow$, then also $u^{\prime} \downarrow$.

PROOF. We limit ourselves to presenting a proof of statement (iii). Assume that $t \preceq u$ and there exists some $t^{\prime}$ such that $t \rightarrow^{k} t^{\prime}$ and $t^{\prime}$ has the variable $x$ as a summand. Let $n>\operatorname{depth}(u)$. Consider the substitution $\sigma$ defined thus:

$$
\sigma(y)= \begin{cases}a^{n+1} \mathbf{0} & \text { if } y=x \\ \mathbf{0} & \text { otherwise. }\end{cases}
$$

Since $t \rightarrow^{k} t^{\prime}$ and $t^{\prime}$ has the variable $x$ as a summand,

$$
\sigma(t) \rightarrow^{k} \sigma\left(t^{\prime}\right) \stackrel{a}{\longrightarrow} a^{n} \mathbf{0} .
$$

As $\sigma(t) \preceq \sigma(u)$, it follows that $\sigma(u) \rightarrow^{k} p \stackrel{a}{\longrightarrow} q$ for some closed terms $p$ and $q$ such that $\sigma\left(t^{\prime}\right) \preceq p$ and $a^{n} \mathbf{0} \preceq q$. By Lemma 8 and statement (i), it follows that $q$ has depth $n$. From $n>\operatorname{depth}(u)$ and the definition of $\sigma$ we conclude that $q$ cannot be of the form $\sigma\left(u^{\prime \prime}\right)$ for any $u^{\prime \prime}$ such that $u \rightarrow^{k+1} u^{\prime \prime}$. (In fact, the depth of such a term $\sigma\left(u^{\prime \prime}\right)$ would either be smaller than $n-k-1$, if $x$ does not occur in $u^{\prime \prime}$, or larger than $n$, if $x$ occurs in $u^{\prime \prime}$.) By the definition of $\sigma$, we may therefore conclude that $q=a^{n} \mathbf{0}$, and $p=\sigma\left(u^{\prime}\right)$ for some $u^{\prime}$ such that $u \rightarrow^{k} u^{\prime}$ and $u^{\prime}$ has $x$ as a summand. Note, moreover, that if $t^{\prime} \downarrow$ then also $\sigma\left(t^{\prime}\right) \downarrow$. As $\sigma\left(t^{\prime}\right) \preceq p=\sigma\left(u^{\prime}\right)$, it follows that $u^{\prime} \downarrow$, which was to be shown.

For each closed term $p$ and $B \subseteq A$, let $B p$ stand for $\sum_{b \in B} b p$. We prove that $\preceq$ is not finitely based by showing that, for any $n \geq 0$, it is impossible to derive the sound inequation

$$
a^{n}(x+A \Omega)+\Omega \preccurlyeq \sum_{b \in A} a^{n}(x+(A \backslash\{b\}) \mathbf{0})+a^{n} A \mathbf{0}
$$

from a set of sound inequations of depth less than $n$. (The depth of an inequation is the depth of its right-hand side.)

Note that for $n=1$ the above inequation generalizes the inequation considered in Example 7. To see that the inequations are sound for all $n \geq 0$, suppose that an arbitrary closed term $p$ is assigned to the variable $x$, and distinguish cases according to whether $\operatorname{depth}(p)=0$ or $\operatorname{depth}(p)>0$. On the one hand, if $\operatorname{depth}(p)=0$, then, modulo $\mathrm{A} 1-\mathrm{A} 4, p=\mathbf{0}$ or $p=\Omega$ and in both cases it is easy to see that $p+A \Omega \preceq A \mathbf{0}$. On the other hand, if $\operatorname{depth}(p)>0$, then there exist $b \in A$ and $p^{\prime}$ such that $p \stackrel{b}{\longrightarrow} p^{\prime}$, and therefore $p+A \Omega \preceq p+(A \backslash\{b\}) \mathbf{0}$.

For convenience of notation we abbreviate the term $\sum_{b \in A} a^{n}(x+(A \backslash\{b\}) \mathbf{0})+a^{n} A \mathbf{0}$ by $R_{n}$.

Proposition 10 Let $E$ be a set of inequations, sound modulo $\preceq$, and let $n$ be a natural number greater than the depth of all the inequations in $E$. Furthermore, let $t$ and $u$ be terms such that $t+\Omega \preceq$ $u+\Omega \preceq R_{n}$ and suppose that $E \vdash t \preccurlyeq u$. If there exists some $t^{\dagger}$ such that $t \rightarrow^{n} t^{\dagger}$ and $x+A \Omega \preceq t^{\dagger} \preceq$ $x+A \mathbf{0}$, then there exists some $u^{\dagger}$ such that $u \rightarrow^{n} u^{\dagger}$ and $x+A \Omega \preceq u^{\dagger} \preceq x+A \mathbf{0}$.

PROOF. We prove the proposition with induction on a derivation of the inequation $t \preccurlyeq u$ from $E$. We distinguish cases, depending on the last rule used in this derivation.

CASE 1: the derivation consists of an application of an axiom in $E$.

Then there exist terms $l$ and $r$ and a substitution $\sigma$ such that $\sigma(l)=t, \sigma(r)=u$ and $l \preccurlyeq r \in$ 
$E$. Now suppose that there exists some $t^{\dagger}$ such that $t \rightarrow^{n} t^{\dagger}$ and $x+A \Omega \preceq t^{\dagger} \preceq x+A \mathbf{0}$. Then $\sigma(l) \rightarrow^{n} t^{\dagger}$ and, since $n>\operatorname{depth}(r) \geq \operatorname{depth}(l)$ by Lemma $9(\mathrm{i})$, there exist $m<n$ and a term $l^{\prime}$ with a variable summand $y$ such that $l \rightarrow^{m} l^{\prime}$ and $\sigma(y) \rightarrow^{n-m} t^{\dagger}$. Hence, since $l \preccurlyeq r$ is sound modulo $\preceq$, by Lemma 9 (iii) there exists a term $r^{\prime}$ with the same variable summand $y$ such that $r \rightarrow^{m} r^{\prime}$. It follows that $u=\sigma(r) \rightarrow^{n} t^{\dagger}$ and, simply according to the assumption, $x+A \Omega \preceq t^{\dagger} \preceq x+A \mathbf{0}$.

CASE 2: the derivation consists of an application of the reflexivity rule.

Then the proposition is immediate.

CASE 3: the last rule applied is the transitivity rule.

Then there exist a term $v$ and derivations of $t \preccurlyeq v$ and $v \preccurlyeq u$ that are proper subderivations of the derivation of $t \preccurlyeq u$. If there exists some $t^{\dagger}$ such that $t \rightarrow^{n} t^{\dagger}$ and $x+A \Omega \preceq t^{\dagger} \preceq x+A \mathbf{0}$, then by the induction hypothesis there exists some $v^{\dagger}$ such that $v \rightarrow^{n} v^{\dagger}$ and $x+A \Omega \preceq v^{\dagger} \preceq x+A \mathbf{0}$. Hence, again by the induction hypothesis, there exists some $u^{\dagger}$ such that $u \rightarrow^{n} u^{\dagger}$ and $x+A \Omega \preceq$ $u^{\dagger} \preceq x+A 0$.

CASE 4: the last rule applied is the congruence rule for $a$.

Then there exist terms $t^{\prime}$ and $u^{\prime}$ such that $t=$ $a t^{\prime}$ and $u=a u^{\prime}$, and a derivation of the inequation $t^{\prime} \preccurlyeq u^{\prime}$ that is a proper subderivation of the derivation of $t \preccurlyeq u$. Now suppose that there exists some $t^{\dagger}$ such that $t \rightarrow^{n} t^{\dagger}$ and $x+A \Omega \preceq t^{\dagger} \preceq$ $x+A \mathbf{0}$. Then, by Lemma 9(iii), $t^{\dagger}$ has the variable $x$ as a summand and moreover it holds that $t^{\dagger} \downarrow$. So, by Lemma 9(iii), there exists some $u^{\dagger}$ such that $u \rightarrow^{n} u^{\dagger}, u^{\dagger}$ also has the variable $x$ as a summand and $u^{\dagger} \downarrow$. Since $u+\Omega \preceq R_{n}$ by the proviso of the proposition, it follows by Lemma 9 (i) that $\operatorname{depth}(u) \leq n$, and hence $\operatorname{depth}\left(u^{\dagger}\right) \leq 1$. Moreover, also from $u+\Omega \preceq R_{n}$ it follows by Lemma 9 (iii) that $x$ is the only variable with an occurrence in $u^{\dagger}$. So $u^{\dagger}=x+B_{1} \Omega+B_{2} \mathbf{0}$ for some $B_{1}, B_{2} \subseteq A$. Therefore, to establish that $x+A \Omega \preceq$ $u^{\dagger} \preceq x+A 0$, it remains to prove that $\mathcal{I}\left(u^{\dagger}\right)=\bar{A}$. To this end, consider the substitution $\sigma$ defined by $\sigma(y)=\mathbf{0}$ for each variable $y$. Note that from $\sigma\left(a t^{\prime}\right)+\Omega \preceq \sigma\left(a u^{\prime}\right)+\Omega \preceq \sigma\left(R_{n}\right)$ and the definition of $R_{n}$ it follows that either there exists some $b \in A$ such that $\sigma\left(t^{\prime}\right) \preceq \sigma\left(u^{\prime}\right) \preceq a^{n-1}(\sigma(x)+$
$(A \backslash\{b\}) \mathbf{0})$ or $\sigma\left(t^{\prime}\right) \preceq \sigma\left(u^{\prime}\right) \preceq a^{n-1} A \mathbf{0}$. However, the former possibility is immediately excluded, for $\sigma\left(t^{\prime}\right) \rightarrow^{n-1} \sigma\left(t^{\dagger}\right)$ and $\mathcal{I}\left(\sigma\left(t^{\dagger}\right)\right)=A$, and hence $\sigma\left(t^{\dagger}\right) \npreceq \sigma(x+(A \backslash\{b\}) \mathbf{0})$. Therefore, it suffices to consider the latter possibility, i.e., $\sigma\left(t^{\prime}\right) \preceq \sigma\left(u^{\prime}\right) \preceq$ $a^{n-1} A$ 0. Since $\sigma\left(u^{\prime}\right) \rightarrow^{n-1} \sigma\left(u^{\dagger}\right)$ it follows that $\sigma\left(u^{\dagger}\right) \preceq A \mathbf{0}$, and hence $\mathcal{I}\left(\sigma\left(u^{\dagger}\right)\right)=A$, as $\sigma\left(u^{\dagger}\right) \downarrow$. From the definition of $\sigma$ it now also follows that $\mathcal{I}\left(u^{\dagger}\right)=A$.

CASE 5: the last rule applied is the congruence rule for + .

Then $t=t_{1}+t_{2}$ and $u=u_{1}+u_{2}$, and there exist derivations of the inequations $t_{1} \preccurlyeq u_{1}$ and $t_{2} \preccurlyeq u_{2}$ that are proper subderivations of the derivation of $t \preccurlyeq u$. Now suppose that there exists some $t^{\dagger}$ such that $t \rightarrow^{n} t^{\dagger}$ and $x+A \Omega \preceq t^{\dagger} \preceq x+A \mathbf{0}$. Then, since $n$ is positive, $t_{1} \rightarrow^{n} t^{\dagger}$ or $t_{2} \rightarrow^{n} t^{\dagger}$. In the first case, since $u_{1}+u_{2}+\Omega \preceq R_{n}$ by the proviso of the proposition and $t_{1} \preceq u_{1}$ by the soundness of $E$, it follows that $t_{1}+\Omega \preceq u_{1}+\Omega \preceq u_{1}+u_{2}+\Omega \preceq R_{n}$. By the induction hypothesis, we have that there exists some $u^{\dagger}$ such that $x+A \Omega \preceq u^{\dagger} \preceq x+A \mathbf{0}$, and clearly $u \rightarrow^{n} u^{\dagger}$ because $n$ is positive. The second case is similar.

Theorem 11 The inequational theory of $B C C S_{\Omega}$ modulo $\preceq$ is not finitely based.

PROOF. Let $E$ be a finite set of inequations over $\mathrm{BCCS}_{\Omega}$ that is sound modulo $\preceq$. Then, by Proposition 10, for $n$ greater than the depths of all the inequations in $E$, the sound inequation

$$
a^{n}(x+A \Omega)+\Omega \preccurlyeq \sum_{b \in A} a^{n}(x+(A \backslash\{b\}) \mathbf{0})+a^{n} A \mathbf{0}
$$

cannot be derived from $E$. Indeed, $a^{n}(x+A \Omega)+$ $\Omega \rightarrow^{n} x+A \Omega \preceq x+A \mathbf{0}$. On the other hand, if $R_{n} \rightarrow^{n} u^{\dagger}$ then either $u^{\dagger}=x+(A \backslash\{b\}) \mathbf{0}$ for some $b \in A$, or $u^{\dagger}=A \mathbf{0}$. In the former case, $x+A \Omega \preceq u^{\dagger}$ fails by Lemma 9 (ii). In the latter case, $x+A \Omega \preceq u^{\dagger}$ fails by Lemma 9(iii).

It follows that every finite set of inequations $E$ that is sound modulo $\preceq$ is necessarily incomplete, and hence $\mathrm{BCCS}_{\Omega}$ modulo $\preceq$ is not finitely based.

Remark 12 The proviso that $A$ be non-empty is necessary for Theorem 11 to hold. In fact, if $A$ were empty, a complete axiomatization for prebisimilarity could be obtained by adding the inequation $x \preccurlyeq \mathbf{0}$ 
to $\mathcal{E}$. Indeed, in that case, it is easy to see that a fully abstract model for $B C C S_{\Omega}$ modulo prebisimilarity can be obtained by considering the two-point domain $\Omega \preccurlyeq \mathbf{0}$.

\section{References}

[1] S. Abramsky. A domain equation for bisimulation. Information and Computation, 92:161-218, 1991.

[2] L. Aceto, W. Fokkink, A. Ingolfsdottir, and M. Mousavi. Lifting non-finite axiomatizability results to extensions of process algebras. Technical Report CSR-08-05, TU/Eindhoven, February 2008.

[3] L. Aceto and M. Hennessy. Termination, deadlock and divergence. Journal of the ACM, 39(1):147-187, 1992.

[4] L. Aceto and A. Ingolfsdottir. CPO models for compact GSOS languages. Information and Computation, 129(2):107-141, 1996.

[5] L. Aceto and A. Ingolfsdottir. A characterization of finitary bisimulation. Information Processing Letters, 64(3):127-134, 1997.

[6] B. Bloom, S. Istrail, and A. Meyer. Bisimulation can't be traced. Journal of the ACM, 42(1):232-268, 1995.

[7] M. Hennessy. A term model for synchronous processes. Information and Control, 51(1):58-75, 1981.

[8] M. Hennessy. Algebraic Theory of Processes. MIT Press, Cambridge, Massachusetts, 1988.

[9] M. Hennessy and G.D. Plotkin. A term model for CCS. In P. Dembiński, editor, $9^{\text {th }}$ Symposium on Mathematical Foundations of Computer Science, volume 88 of Lecture Notes in Computer Science, pages 261-274. Springer-Verlag, 1980.

[10] J.F. Groote. New strategy for proving $\omega$-completeness applied to process algebra. In J.C.M. Baeten and J.W. Klop, editors CONCUR '90, Theories of Concurrency: Unification and Extension, volume 458 of Lecture Notes in Computer Science, pages 314-331, Spinger-Verlag, 1990.

[11] R.J. van Glabbeek and W.P. Weijland. Refinement in branching time semantics. In Proceedings of AMAST Conference, pages 197-201, Iowa City, USA, 1989.

[12] R. Milner. Fully abstract models of typed $\lambda$-calculi. Theoretical Computer Science, 4:1-22, 1977.

[13] R. Milner. A modal characterisation of observable machine behaviour. In E. Astesiano and C. Böhm, editors, CAAP '81: Trees in Algebra and Programming, 6th Colloquium, volume 112 of Lecture Notes in Computer Science, pages 25-34. Springer-Verlag, 1981.

[14] R. Milner. Communication and Concurrency. PrenticeHall International, Englewood Cliffs, 1989.

[15] G.D. Plotkin. LCF considered as a programming language. Theoretical Computer Science, 5:223-256, 1977.

[16] G.D. Plotkin. A structural approach to operational semantics. Journal of Logic and Algebraic Programming, 60-61:17-139, 2004.

[17] D.J. Walker. Bisimulation and divergence. Information and Computation, 85(2):202-241, 1990. 